\title{
Temporal Lau effect: Noncoherent Regeneration of Periodic Pulse Trains
}

Jesús Lancis

\section{Cristina M. Gómez-Sarabia}

Jorge Ojeda-Castañeda

\section{Carlos R. Fernández-Pousa}

Pedro Andrés
Departament de Física, Universitat Jaume I, 12080 Castelló, Spain

Departament de Física, Universitat Jaume I, 12080 Castelló, Spain

Departament de Física, Universitat Jaume I, 12080 Castelló, Spain

Permanent Address IICO, Autonomous University of San Luis Potosí, México

Teoría de la Señal y Comunicaciones, Universidad Miguel Hernández, Elche 03202, Spain

Departamento de Óptica, Universitat de València, 46100 Burjassot, Spain

We present an optical method for implementing the temporal Talbot effect with a spectrally incoherent optical source and a linear dispersive medium, at the first-order dispersion regime. We state the condition for achieving this effect, here denoted as the temporal Lau effect. [DOI: $10.2971 /$ jeos.2006.06018]

Keywords: Temporal Talbot effect, dispersive media, multiple-wavelength sources, optical pulse propagation, temporal Lau effect

\section{I n trod uct io n}

The temporal Talbot effect allows regenerating a periodic pulse train, which is launched into dispersive single-mode optical fibers [1]-[3], chirped fiber gratings [4], and multimode optical fibers [5].

This temporal regeneration effect also finds useful applications for suppressing dispersion effects in pulsed fiber lasers [6], or for tailoring the repetion rate and the duty cycle of linearly chirped signals [7].

However, despite its usefulness, the temporal Talbot effect strongly depends on the source linewidth [8, 9]. Hence, one is tempted to assume that the temporal Talbot effect can only be implemented with spectrally narrow sources. Here we consider certain multi-wavelength optical sources, which are of interest in time-resolved spectroscopy, optical component characterization, and in wavelength-divisionmultiplexed WDM communications. These sources can be implemented either as an array of distributed-feedback (DFB) laser diodes (LDs) or by means of a single laser emitting simultaneously at different wavelengths $[10,11]$.

Our aim is to present an optical method that uses a spectrally incoherent optical source, which is periodically modulated in intensity by means of an external modulator (EM), e.g., electroabsorption or electrooptic, for implementing temporal regeneration of periodic pulse trains in a linear dispersive medium. In the framework of the space-time analogy, our proposal can be thought of as the temporal counterpart of the Lau effect [12], or the noncoherent Talbot effect [13].
To our end, in section 2, we identify certain multi-wavelength power spectra. In section 3 we show that, in a group-delaydispersion (GDD) circuit, the novel power spectrum sustains the polychromatic temporal Talbot effect provided that a frequency condition is satisfied. In section 4 , we discuss the influence of a mismatch in the frequency condition. And in section 5 , we summarize our proposal.

\section{M U LTI-W AVELENGTH SOURCES}

We propose to use the following spectrally incoherent optical source. Its power spectrum is composed by $(2 \mathrm{M}+1)$ narrow spectral lines.

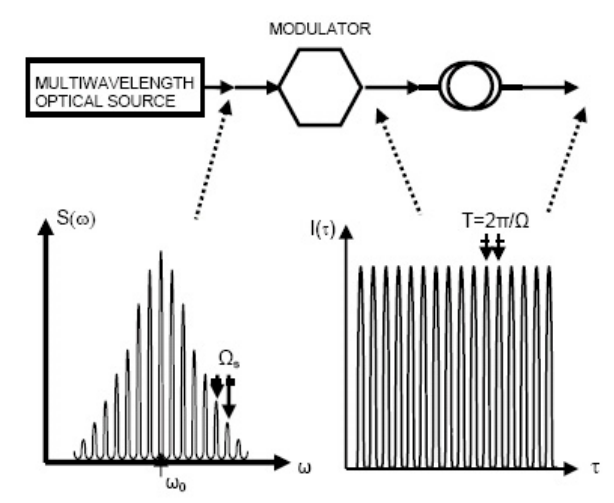

FIC. 1 Schematic diagram of the proposed optical setup. For this picture we assume a Gaussian-modulated spectrum for the multi-wavelength optical source and a Gaussiantype modulating signal. 
Each spectral line is located at $\omega=\omega_{0}+m \Omega_{S}$, where $\omega_{0}$ is the source central angular frequency, $m$ is an integer number, and $\Omega_{S}$ is an angular frequency to be specified in what follows. As is depicted in Figure 1, if the spectral lines are symmetrically located around $\omega_{0}$, then the normalized source power spectrum is

$$
S(\omega)=\sum_{m=-M}^{+M} S_{m} \delta\left(\omega-\omega_{0}-m \Omega_{S}\right) .
$$

In Eq.(1) $S_{m}$ denotes the spectral intensity of the m-th spectral channel. We assume that the dispersion relation can be approximated with a Taylor series expansion, around $\omega_{0}$, for any frequency $\omega$ within the interval $\left(\omega_{0}-M \Omega_{S}, \omega_{0}+M \Omega_{S}\right)$. This assumption is further discussed in the next section.

We consider that the spectrally incoherent source is modulated by an EM, which is driven with a periodic RF signal. In this way, at the input of the optical fiber, the slowly varying complex-amplitude envelope is represented by the Fourier series

$$
g(t)=\sum_{n=-\infty}^{+\infty} a_{n} \exp [i n \Omega t]
$$

where we denote as $\Omega=2 \pi / T$ the angular frequency of the driving signal and, of course, of the pulse train. The Fourier spectrum of the slowly varying complex-amplitude envelope is

$$
G(\omega)=\sum_{n=-\infty}^{+\infty} a_{n} \delta(\omega-n \Omega)
$$

The above signal is then propagated through a GDD circuit. From now on, and with no lack of generality, we assume the GDD circuit is implemented by means of single-mode optical fiber.

\section{M ULTI-W A VELENGTH PERI- ODICPULSE PROPAGATION}

Following the method adopted by Wang [14], the field propagation a distance $\mathrm{z}$ along fiber is described through the convolution integral

$$
\begin{array}{r}
R(t, z, \omega)=\int_{-\infty}^{+\infty} G\left(\omega^{\prime}-\omega\right) \exp \left[i \beta(\omega) z-i \omega^{\prime} t\right] d \omega^{\prime}= \\
\exp (-i \omega t) \sum_{n=-\infty}^{+\infty}\left\{a_{n} \exp [i \beta(\omega-n \Omega) z]\right\} \exp (i n \Omega t) .
\end{array}
$$

In Eq.(4) we denote as $\beta(\omega)$ the dispersion relation. We consider that, at the output of the fiber, the detected output signal is proportional to the ensemble average of the square modulus of the slowly varying envelope. That is,

$$
I(t, z)=(1 / 2 \pi) \int_{-\infty}^{+\infty} S(\omega)|R(t, z, \omega)|^{2} d \omega .
$$

By substituting Eq.(1) in Eq. (5) we have that

$$
I(t, z)=(1 / 2 \pi) \sum_{m=-M}^{+M} S_{m}\left|R\left(t, z, \omega_{0}+m \Omega_{S}\right)\right|^{2} .
$$

Furthermore by substituting Eq.(4) in Eq.(6) we obtain

$$
\begin{array}{r}
I(t, z)=(1 / 2 \pi) \sum_{m=-M}^{+M} S_{m} \sum_{p=-\infty}^{+\infty} \sum_{q=-\infty}^{+\infty} a_{p} a_{q}^{*} \exp [i(p-q) \Omega t] \times \\
\exp \left[i \beta\left(\omega_{0}+m \Omega_{S}-p \Omega\right) z-i \beta\left(\omega_{0}+m \Omega_{S}-q \Omega\right) z\right] .(7)
\end{array}
$$

It is convenient to rewrite Eq.(7), in terms of a new integer variable $n=p-q$, as

$$
\begin{aligned}
& I(t, z)=(1 / 2 \pi) \sum_{m=-M}^{+M} S_{m} \sum_{n=-\infty}^{+\infty} \sum_{q=-\infty}^{+\infty} a_{n+q} a_{q}^{*} \exp [i n \Omega t] \times \\
& \exp \left[i \beta\left(\omega_{0}+m \Omega_{S}-q \Omega-n \Omega\right) z-i \beta\left(\omega_{0}+m \Omega_{S}-q \Omega\right) z\right] .
\end{aligned}
$$

Next, we state the assumptions made for approximating the dispersion relation as the following Taylor series expansion

$$
\beta(\omega)=\beta_{0}+\beta_{1}\left(\omega-\omega_{0}\right)+\frac{\beta_{2}}{2}\left(\omega-\omega_{0}\right)^{2},
$$

where $\beta_{n}=(d / d \omega)^{n} \beta(\omega)$, at $\omega=\omega_{0}$. For a typical optical fiber operating at the third telecommunications window $\left(\lambda_{0}=1.55 \mu \mathrm{m}\right), \beta_{2}=-21.6 \mathrm{ps}^{2} / \mathrm{km}$ and the third-order dispersion (TOD) coefficient is $\beta_{3}=0.127 \mathrm{ps}^{3} / \mathrm{km}$. This means that the maximum bandwidth of the optical source, $2 M \Omega_{S}$, must be kept much lower than $80 \mathrm{THz}$ to ensure negligible effect from the TOD term in the Taylor expansion. From a practical point of view, for a 100 channel WDM network, the above condition is satisfied for a spectral spacing of $\Omega_{S}=0.8 \mathrm{THz}$ $(\Delta \lambda=6.4 \mathrm{~nm})$.

From Eqs.(8) and (9) we have that

$$
\begin{gathered}
I(\tau, z)=(1 / 2 \pi) \sum_{m=-M}^{+M} S_{m} \sum_{n=-\infty}^{+\infty} \sum_{q=-\infty}^{+\infty} a_{n+q} a_{q}^{*} \times \\
\exp \left[i n \Omega\left(\tau-\beta_{1} z\right)\right] \exp \left[i\left(2 m \frac{\Omega_{S}}{\Omega}+2 q+n\right) \frac{n \beta_{2} \Omega^{2} z}{2}\right] .
\end{gathered}
$$

In the first-order dispersion regime, we note that for achieving temporal Talbot effect (neither dispersion, nor shifts) the distance $\mathrm{z}$ should be a multiple integer number of the so-called Talbot distance, $z_{T}=4 \pi / \beta_{2} \Omega^{2}$. At multiples of this distance, say at $z=L z_{T}$ with $L=1,2,3 \ldots$, and using the proper time, $\tau=t-\beta_{1} z$, Eq.(10) reduces to

$$
\begin{gathered}
I\left(\tau, L z_{T}\right)=(1 / 2 \pi) \sum_{n=-\infty}^{+\infty} \sum_{q=-\infty}^{+\infty} a_{n+q} a_{q}^{*} \times \\
\sum_{m=-M}^{+M} S_{m} \exp \left[i 2 \pi \operatorname{Ln}\left(\frac{\tau}{T}+m \frac{\Omega_{S}}{\Omega}\right)\right] .
\end{gathered}
$$

Or equivalently, from $|g(t)|^{2}$ in Eq.(2),

$$
I\left(\tau, L z_{T}\right)=(1 / 2 \pi) \sum_{m=-M}^{+M} S_{m}\left|g\left(\tau+L m T \frac{\Omega_{S}}{\Omega}\right)\right|^{2} .
$$

It is apparent from Eq.(12) that the output signal for a fiber length corresponding to a multiple of the Talbot distance corresponds to a set of multiple weighted and shifted selfimages of the intensity driving signal $|g(t)|^{2}$. Furthermore, the amount of shift is different for each channel. This fact prevents in general from obtaining temporal regeneration of the input signal when a multi-wavelength optical source is employed.

However, here we show that there is a constant time delay $\Delta \tau=L\left(\Omega_{S} / \Omega\right) T$ between successive channels. In this way, 
we claim that it is possible to implement the temporal Talbot effect with a multi-wavelength optical source if $\Delta \tau$ is set to a multiple of the driving period $T$. In mathematical terms, and for the first self-image distance $L=1$, the following frequency condition must be satisfied

$$
\Omega_{S}=Q \Omega,
$$

where $Q$ is an integer number. The condition in Eq.(13) is denoted as the temporal Lau condition. We note, for the first time, that this condition is the temporal analogue of the consonance condition required in the spatial domain to achieve high-contrast Lau fringes, with an extended spatially incoherent optical source $[12,13]$. The role of the periodic input source in the spatial domain is here played by the multi-wavelength optical source. Note that the frequency detuning $\Omega_{S}$ is the temporal counterpart of the spatial period of the incoherent source in the Lau experiment.

Next, we consider the feasibility of the practical implementation. To our goal, we assume a WDM scheme at the third telecommunications window where the multi-wavelength source is implemented by means of an array of continuous wave DFB lasers. We assume a channel spacing $f_{s}=\Omega_{s} / 2 \pi=$ $100 \mathrm{GHz}, \Delta \lambda=0.805 \mathrm{~nm}$, and, for the analysis, we consider a 10 channel scheme. In accordance with Eq.(13), the noncoherent temporal self-image at the first self-image distance, $\mathrm{L}=1$, will be achieved whenever the driving signal is set to $f=\Omega / 2 \pi=(100 / Q) G H z$. For $Q=10$, this corresponds to a feasible $10 \mathrm{GHz}$ RF signal. Note the frequency signal requirement can be reduced either by choosing a higher value for the integer $Q$ or lower channel spacing as in a DWDM scheme.

\section{M ISMATCH IN THE LAU CONDI- T I 0 N}

Finally, we discuss the impact of some mismatch in the temporal Lau condition. That is

$$
\Omega_{S}=(Q+\varepsilon) \Omega
$$

where $\varepsilon$ is a real positive number, such that $0<\varepsilon<1$. Using the previous formulation, it is straightforward to obtain that the output irradiance is now

$$
\begin{gathered}
I\left(\tau, L z_{T}\right)=(1 / 2 \pi) \sum_{m=-M}^{+M} S_{m}|g(\tau+L m \varepsilon T)|^{2}= \\
(1 / 2 \pi) \sum_{n=-\infty}^{+\infty} c_{n} \exp [i n \Omega \tau] \sum_{m=-M}^{+M} S_{m} \exp [i 2 \pi L m n \varepsilon] .
\end{gathered}
$$

where $c_{n}$ denotes the autocorrelation coefficient of the Fourier components $a_{n}$.

It is apparent from Eq.(15) that a mismatch, in the temporal Lau condition, generates a linear phase delay between the Fourier components, which may be related to the discrete Fourier transform of the source power spectrum. However, this is beyond our present scope. Here we only analyze two particular cases that illustrate the main features of our proposal.
If we set $L=1, \varepsilon=1 / 2$ and the coefficients of the power spectrum are $S_{0}=S_{1}$ and zero otherwise, then Eq.(15) becomes

$$
I\left(\tau, z_{T}\right)=\left(S_{0} / 2 \pi\right)\left\{|g(\tau)|^{2}+|g(\tau+T / 2)|^{2}\right\} .
$$

Hence, for a periodic, rectangular pulse train with duty cycle equal to one half, $c_{2 n}=0$, one has that

$$
|g(\tau+T / 2)|^{2}=1-|g(\tau)|^{2} .
$$

And thus, Eq.(16) predicts zero modulation at the output. That is, $I\left(\tau, z_{T}\right)=\left(S_{0} / 2 \pi\right)$, the irradiance distribution is uniform.

In the second example, we consider that the coefficients of the power spectrum have the same value, $S_{m}=S_{0}=1 /(2 M+1)$ for any $m$. Then, Eq.(15) becomes

$$
I\left(\tau, z_{T}\right)=\frac{1}{2 \pi(2 M+1)} \sum_{m=-M}^{+M}|g(\tau+L m \varepsilon T)|^{2} .
$$

This irradiance profiles are shown in Figure 2 and Figure 3, for a periodic pulse, with duty cycle equals to $1 / 4$.

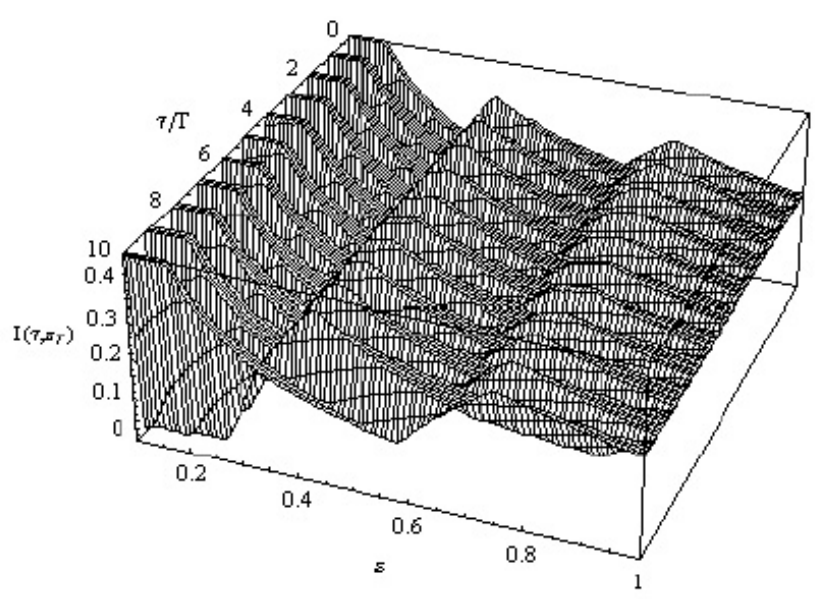

FIG. 2 Normalized irradiance variations, of the temporal Lau effect, with 3 spectral lines. The mismatch, parameter, $\varepsilon$, varies from 0.1 to 0.9 .

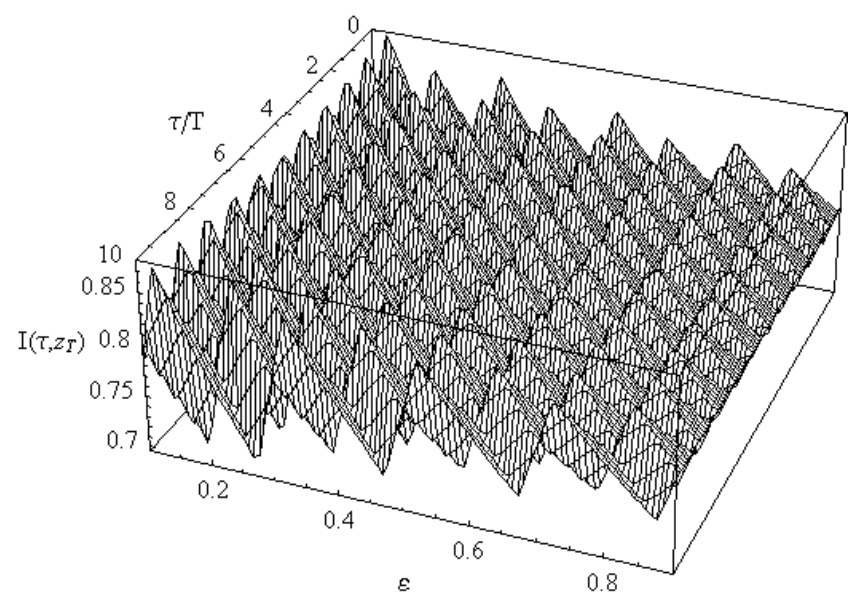

FIG. 3 Same as Figure 2, but for 11 spectral lines.

The parameter $\varepsilon$ varies from 0.1 till 0.9. In Figure 2 the number of incoherent lines sources is $3=2 M+1$, or $M=1$; 
while in Figure 3 the number of incoherent lines sources is $11=2 M+1$, or $M=5$. We note that for this example, the visibility of the irradiance distribution decreases as the parameter $\varepsilon$ increases.

\section{C O N CLUSIONS}

Summarizing, we have shown that with a suitable multiwavelength optical source, it is possible to implement the temporal Talbot effect; here denoted as the temporal Lau effect. Then, one can use a spectrally incoherent source for regenerating a slow varying periodic pulse train, which is launched into a linear dispersive (lowest-dispersion-order) optical fiber. For first time, we have identified the condition for implementing the temporal Lau effect. And we have discussed the impact of a mismatch in the Lau condition.

\section{ACKNOWLEDG M E N T}

This research was funded by the Dirección General de Investigación Científica y Técnica, Spain, and FEDER, under the project FIS2004-02404 and TIC2003-03041. J.O.-C. gratefully acknowledges the financial support from "Convenio UJIBancaixa" (Grant 06I005.27).

\section{References}

[1] T. Janson and J. Janson, "Temporal self-imaging effect in singlemode optical fiber" J. Opt. Soc. Am. 71, 1373-1376 (1981).

[2] P. A. Belanger, "Periodic restoration of pulse trains in a linear dispersive medium" IEEE Photon. Technol. Lett. 1, 71-72 (1989).
[3] G. Indebetouw, "Necessary condition for temporal self-imaging" J. Mod. Opt. 37, 1439-1451 (1990).

[4] J. Azaña and M. A. Muriel, "Temporal Talbot effect in fiber gratings and its applications" Appl. 0pt. 38, 6700-6704 (1999).

[5] V. P. Minkovich, A. N. Starodumov, V. I. Bosirov, V, I, Lebedev, S. N. Perepechko, "Temporal interference of coherent laser pulses in optical fibers" Opt. Commun. 192, 231-235 (2001).

[6] B. Fischer, B. Vodonos, S. Atkins, A. Bekker, "Dispersion-mode pulsed laser" Opt. Lett. 25, 728-730 (2000).

[7] J. Lancis, J. Caraquitena, P. Andrés and M. A. Muriel, “Temporal Self-Imaging Effect for chirped laser pulse sequences: Repetion rate and duty cycle tunability" Opt. Comm. 253, 156-163 (2005).

[8] A. M. Vengsarkar and I. M. Besieries, “Regenerative Periodic Pulse Trains in Linear dispersive, single-mode Optical fibers: Effects of Finite Source Linewidth" J. Lightwave technol. 3, 33-35 (1991).

[9] Laura Chantada, Carlos R. Fernández-Pousa and Carlos CómezReino, "Theory of the partially coherent temporal Talbot effect" Opt. Comm. 266, 393-398 (2006).

[10] T. Morioka, K. Mori, S. Kawanishi, and M. Saruwatari, “Multi-WDMchannel, Gbit/s pulse generation from a single laser source utilizing LD-pumped supercontinuum in optical fibers" IEEE Photonics Technol. Lett. 6, 365-367 (2006).

[11] C. Shu and S: P. Yam, "Effective generation of tunable single- and multi-wavelength optical pulses from a Fabry-Perot laser diode" IEEE Photonics Technol. Lett. 9, 1214-1216 (2006).

[12] J. Jahns and A. W. Lohmann, "Lau effect: a diffraction experiment with incoherent illumination" Opt. Comm. 28, 263-266 (1979).

[13] J. Ojeda-Castaneda, J. Ibarra and J. C. Barreiro, "Noncoherent Talbot effect: coherence theory and applications" Opt. Comm. 71, 151-155 (1989).

[14] C. Wang, "Transmission of a Gaussian pulse in a single mode fiber system" J. Lightwave Technol. 1, 572-579 (1983). 